Revue d'histoire de l'Amérique française

REVUE D.HISTOIRE DE L'AMÉRIQUE FRANÇAISE

\title{
Le voyage d'André Michaux au lac Mistassini en 1792
}

\section{Jacques Rousseau}

Volume 2, numéro 3, décembre 1948

URI : https://id.erudit.org/iderudit/801477ar

DOI : https://doi.org/10.7202/801477ar

Aller au sommaire du numéro

Éditeur(s)

Institut d'histoire de l'Amérique française

ISSN

0035-2357 (imprimé)

1492-1383 (numérique)

Découvrir la revue

Citer cet article

Rousseau, J. (1948). Le voyage d'André Michaux au lac Mistassini en 1792.

Revue d'histoire de l'Amérique française, 2(3), 390-423.

https://doi.org/10.7202/801477ar d'utilisation que vous pouvez consulter en ligne.

https://apropos.erudit.org/fr/usagers/politique-dutilisation/ 


\title{
LE VOYAGE D'ANDRÉ MICHAUX AU LAC MISTASSINI EN 1792
}

\author{
Les Premiers voyages au lac Mistassini
}

Aux seizième et dix-septième siècles, l'un des objectifs des voyageurs français dans le nord-est de l'Amérique était, avec la traite des fourrures, la découverte d'un passage vers la mer de l'Ouest; mais une puissance étrangère leur coupa bientôt la route au nord. Les erreurs du gouvernement de la Nouvelle-France, - aggravées sans doute par le caractère de DesGroseilliers et de Radisson, avaient poussé ces commerçants de fourrures vers l'Angleterre et provoqué l'organisation de la Compagnie des Aventuriers de la Baie d'Hudson.

Quand les Anglais s'installèrent pour de bon à la baie d'Hudson, qui paraissait l'étape normale vers la mer de l'Ouest, - les Français cherchèrent à ruiner leur commerce en attirant vers eux les Indiens qui y faisaient la traite.

Ce fut l'une des raisons des quelques expéditions qui tendirent vers la Baie, dès le dix-septième siècle. La plupart passèrent par le lac Mistassini. Guillaume Couture, en 1663, se rend au lac Nemiskau, à mi-chemin entre le lac Mistassini et la baie James. ${ }^{1}$ En 1672, le P. Charles Albanel et Paul Denis de Saint-Simon ${ }^{2}$ atteignent la Baie.

1. Ce voyage est relaté dans le manuscrit suivant: Enqueste faite par le lieutenant général de la Prévosté de Québec (1688). Archives canadiennes (Ottawa), C. $11 \mathrm{~A}$, vol. 10, p. 165-173. Voir aussi Delanglez, Jean, The voyage of Louis Jolliet to Hudson Bay in 1679. Mid-America, 26:221-250. (July 1944). Le récit d'un voyage antérieur de Couture à la baie James, raconté par Joseph-Édouard Roy, dans "Guillaume Couture, premier colon de la Pointe Lévy " (Lévis, 1884), était basé sur un faux. Voir à ce sujet Delanglez, op. cit.

2. Le journal du Père Albanel est inclus dans la relation du Père Dablon de 1671-1672. (Relations des Jésuites, ed. Thwaites, 56:148-217. 1899). La relation de Saint-Simon est dans l' " Enqueste faite par le lieutenant génłéral de la Prévosté de Québec " (1688), précédemment citée. Voir aussi Rousse a , Madeleine et Jacques, La Crainte des Iroquois chez les Mistassins, Revue d'histoire de l'Amérique française, 2: 13-26. 1948. 
Le Père de Crépieul ${ }^{3}$, en 1674, évangélise les Mistassins. Louis Jolliet se rend à la baie James, en passant par le lac Mistassini, en $1679^{4}$. $\mathrm{Au}$ début du dix-huitième siècle, Joseph Dorval réorganise la traite au lac Mistassini. ${ }^{5}$ Depuis, il y a toujours eu là un poste ou des traiteurs, relevant d'abord des Postes du Roi, puis de la Compagnie du Nordouest, puis de la Compagnie de la baie d'Hudson, au début du siècle dernier. Ces deux dernières compagnies demeurèrent concurremment au lac Mistassini de 1800 jusqu'à 1821, époque où la H. B. Co. absorba sa rivale. ${ }^{6}$

C'est dire que les voyageurs français fréquentent assez régulièrement le lac Mistassini, depuis 1663 jusqu'aux premières années du dix-neuvième siècle, car la compagnie du Nord-ouest emploje fréquemment des commis canadiens-français. Les traiteurs n'ont apparemment pas laissé de récits détaillés de leurs voyages. Pour eux, la géographie s'apprenait sur le terrain, lorsque, jeunes apprentis, ils s'adjoignaient aux équipes des vétérans. Les documents relatifs aux voyages de Guil-

3. Le journal du Père de Crépieul est inclus dans la relation du Père Dablon de 1673 à 1674. (Relations des Jésuites, ed. Thwajtes, 59: 24-53. 1900).

4. Voir Delanglez, op. cit. Mid-America, 26: 221-250. 1944. Voir aussi cartes de Jolliet 1679 (trois variantes) et 1684. L'une des cartes de 1679 est reproduite dans Pin art, A.-L. Recueil de cartes, plans et vues relatifs aux Êtats-Unis et au Canada, Paris 1893. Voir également DelangLez, Life and voyages of Louis Jolliet (1645-1700). 289 p., Institute of Jesuit History publications, Chicago 1948. En appendice, (p. 252-257), se trouve l'étude suivante: "Voyage de Jolliet à la baie d'Hudson " par Jacques Roussead. La découverte postérieure de documents de base m'oblige à corriger en partie mes vues. S'il est vrai que Jolliet est bien passé par la Péribonka pour l'un des trajets, comme je ie croyais, par contre, contrairement à l'opinion que j'ai émise, il est également passé, pour l'autre trajet, par la rivière Miistassini.

5. Mémoire sur toutes les parties de la régie du Domaine d'Occident en Canada. 1732, Archives des Colonties, Paris, C"A, 59-2. Copie aux Archives canadiennes, Ottawa; aussi copie photographique et copie au clavigraphe; Société historique du Saguenay, Chicoutimi, Doc. 28 et Doss. 308: "L'hyvernement des Mistassins est rétably depuis quatre ou cinq ans. Joseph Dorval, engagé, y a découvert... " Le " rétablissement " de la traite daterait donc des environs de 1727 ou 1728 et les Français y auraient fait la traite antérieurement. La " maison Jolliet " de la carte de Jolliet de 1684 et la [ maison des ] "françois " de la carte de Jolliet de 1679, pourraient être le premier poste régulier de traite établi dans ces parages. (Il n'existe aucune preuve toutefois que ce fut un poste de traite). Auparavant, en 1663, Guillaume Couture avait fait la traite, comme en témoigne l'" Enqueste... " de 1688. (Pour référence, voir note 1): "Il se crut obligé de ne pas passer plus outre et après avơir nlegocié avec eux et leur avoir fait des présens pour une alliance future... " - Au sujet des Dorval, voîr aussi " l'Histoire du Saguenay " (Publ. Soc. historique du Saguenay, no 3), p. 127-128, Chicoutimi 1938.

6. Voorhis, Ernest, Historic Forts and Trading Posts of the French Regime and of the English Fur Trading Companies. 1930. 
laume Couture, de Louis Jolliet, des pères Albanel et de Crépieul, ne renferment que des notes très imprécises sur le trajet suivi. Pour le voyage de Jolliet, toutefois, une réserve s'impose: si le seul récit qu'on en connaît ne nous renseigne guère, les cartes de son voyage, par contre, sont plus éloquentes. ${ }^{7}$

Le botaniste français André Michaux, ${ }^{7 a}$ venu en Amérique en 1785 pour y récolter des plantes pour le Jardin du Roi, et que la Révolution Française obligea à prolonger son séjour, décida en 1792 de se rendre à la baie d'Hudson par le Saguenay, le lac St-Jean, le lac Mistassini et la rivière Rupert. La saison avancée ne lui permit pas toutefois d'exécuter entièrement son projet et il dut rebrousser chemin alors qu'il se trouvait encore sur la rivière Rupert. Le journal de l'expédition ${ }^{8}$, assez élaboré, se prête beaucoup plus à l'étude que les récits de Couture, Albanel, Crépieul et Jolliet. Contrairement à ses prédécesseurs, Michaux n'est pas nanti d'une mission politique, économique ou évangélique. O'est en naturaliste qu'il voyage et toutes ses pensées sont consacrées à l'herborisation, à l'observation du territoire et à la rédaction de ses notes qu'il consigne tous les soirs dans un cahier à la lueur du feu de camp. On comprend donc que son journal nous permette plus facilement de nous reconnaître dans la mosaïque complexe des lacs et l'écheveau emmêlé des rivières. Bien plus, le journal de Michaux pourra nous permettre éventuellement de mieux interpréter des voyages antérieurs.

7. Voir Delanglez, op. cit. Mid-America, 26: 221-250. 1944. - id. Life and Voyages of Louis Jolliet, op. cit. Rousse AU, Jacques, Voyage de Jolliet à la baie d'Hudson, app. A. de l'ouvrage précédent, op. cit.

7a) Sur André Michaux, voir notamment: Deleuze, Joseph-P. F., Notice historique sur André Michaux. Annales du Museum d'Histoire naturelle (Paris), Vol. 3 191-227. 1804. - LACRoIx Alfred, Notice historique sur les membres et correspondants de l'Academie des Sciences ayant travaillé dans les colonies françaises des Mascareignes et de Madagascar au XVIII $I^{\mathbf{e}}$ siècle et au début du XIXe. Brochure de $119 \mathrm{p}$. Institut de France, Académie des Sciences, 1934. Les pages 101-107 sont consacrées à André Michaux.

8. Michadx, André [ Journal de ] 1792. Ms de 107 p. (y compris les p. 1-2 de la couverture) non numérotées. Archives de l'American Philosophical Society, Philadelphie. Copie photographique dans les collections du Jardin botanique de Montréal. Le journal complet d'André Michaux a été publié par SARGENT: "Journal of André Michaux, 1787-1796, with an introduction and notes by Charles Sprague Sargen+" Proc. Amer. Phil. Soc., vol. 26 (129), 145 p. 1888. Le texte n'est pas toujours absolument conforme au manuscrit. La partie du journal relative au lac Saint-Jean et au lac Mistassini a été reproduite, avec les imperfections du texte de SARGENT, dans: Marie-Victorin, F., Études floristiques sur la région du lac Saint-Jean, Contrib. lab. bot. Univ. Montréal, no 4, p. 23-55. 1925. 


\section{Opinions diverses sur l'itinéraire de Michaux.}

Cela ne signifie pas que le trajet de Michaux n'ait pas fait luimême l'objet de discussions. Dans son journal, le botaniste affirme être monté par la rivière Mistassini, jusqu'au portage de Monte-àpeine, puis avoir atteint, par le lac des Cygnes, une rivière qui coule vers le nord, enfin le lac Mistassini et la Rupert. Les tentatives d'interprétations, - où interviennent trop d'opinions fantaisistes, basées uniquement sur l'examen sommaire de cartes imparfaites, ou ellesmêmes fantaisistes, d'une région encore incomplètement explorée, ont beaucoup contribué à jeter de la confusion dans une histoire relativement claire. Avant de suivre pas à pas Michaux, dans son itinéraire, voyons brièvement les opinions des auteurs qui se sont intéressés au problème.

L'abbé Ovide Brunet, ${ }^{9}$ en 1861 et 1863, publie des opuscules sur le voyage de Michaux à la rivière Rupert. Ayant lui-même examiné, à Paris, les plantes rapportées par le botaniste et copié la partie du journal qui l'intéressait (car il n'avait pas encore été publié alors), il raconte simplement le voyage sans tenter d'explication géographique. Se contentant de résumer l'auteur, il croit comme lui la hauteur des terres dans le voisinage du lac des Cygnes. Opinion à peine esquissée, mais qui ne fait pas de doute lorsqu'on parcourt le texte accompagnant les spécimens de Michaux et que Brunet cite comme croissant à la hauteur des terres.

Le géologue Albert Peter Low, qui se rendit au lac Mistassini en $1884,{ }^{10}$ consacre quelques paragraphes, dans son rapport, ${ }^{11}$ aux voyageurs antérieurs. On y voit que Michaux suivit la rivière Mistassini, prenant " the route taken by Père Albanel, and quotes him for his description. "' Toutefois, l'auteur a oublié de nous dire antérieure-

9. Brunet, Abbé Ovide, Voyage d'André Michaux en Canada depuis le lac Champlain jusqu'à la baie d'Hudson. 27 p., Bureau de l'Abeille, Québec 1861. - Le même. Notice sur les plantes de Michaux et sur son voyage au Canada et à la baie d'Hudson, d'après son journal manuscrit et autres documents inédits, 45 p., Bureau de l'Abeille, Québec 1863. - Le même: Michaux and his journey in Canada, CANA DIAN Naturalist, New Series, 1:331-343. 1864. Une carte (Traduction de T. Sterry-Hunt).

10. Rousseau, Jacques, Bataille de sextants autour du lac Mistassini. Action Universitaire, 14e Année: 99-116 (Janv.) 1948.

11. Low, A.P., Report of the Mistassini Expedition, 1884-1885. Geological and Natural History Survey of Canada, Part D, Annual report, 1885. (Voir p. 12-13). 
ment par où est passé Albanel. Il est cependant d'avis que, " he must have crossed only the southern end [ of Lake Mistassini] on his way to the Rupert River. " Michaux et Albanel, qu'ils aient suivi ou non la rivière Mistassini, auraient donc atteint le grand lac par son extrémité sud-ouest et non par le lac Albanel. Sur quel témojgnage se base Low pour affirmer que Michaux a suivi la route d'Albanel, je l'ignore. Michaux, dans son journal ne mentionne pas non plus le voyage d'Albanel. Il est probable que Low a mal compris un passage de Brunet ${ }^{12}$. D'autre part, dans un autre ouvrage, ${ }^{13}$ Low affirme qu'Albanel s'est rendu au lac Mistassini par l'Ashouapmouchouan. Donc jugements très sommaires, sans valeur critique. D'ailleurs, l'auteur ne se propose aucunement d'analyser les jtinéraires.

Sargent, en publiant le journal manuscrit de Michaux, ${ }^{14}$ ne présente aucune interprétation du trajet. Beaucoup plus sommaire encore que l'opinion de Low, celle d'Henry O'Sullivan. ${ }^{15}$ Michaux fils ${ }^{16}$ ayant affirmé, sur la foi du journal de son père, qu'à la hauteur des terres, entre le lac Saint-Jean et le lac Mistassini, la forêt est réduite à la plus simple expression et ne comprend souvent que des pins rabougris, O'Sullivan rétorque avec indignation: " Mr. Michaud claims to have gone up to Mistassini via Lake St. John and descended the Rupert River to within a short distance of James Bay and returned by the same route. In the interest of the province it is time that such

12. BRUNet, 1861, op. cit.

13. Low, A.P., Report on explorations in the Labrador Peninsula along the East Main, Koksoak, Hamilton, Manicuagan and portions of other rivers in 1892-93-94-95. Geological Survey of Canada, part L., Annual report, vol. 8, 1896. (Voir p. 11). - G.-F. Baillargé, dans un ouvrage que j'ai découvert après la composition typographique de mon étude, affirme lui aussi, mais avant Low, que Michaux a suivi la route du père Albanel. C'est peut-être même là l'origine de l'opinion de Low. Toutefois, contrairement à ce dernier, Baillargé note qu'Albanel est passé par la rivière Mistassini. Voir BaIllargé G.-F., Memoire sur les régions du lac Saint-Jean et du Saguenay, ainsi que sur les travaux exécutés, en voie d'exécution et projetés, $\grave{a}$ differents endroits dans ces régions, accompagné de notes sur la baie d'Hudson et sur la navigation de cette baie, etc., etc. Annexe no 8 (p. 343-446) de: Rapport sur les travaux publics, 1868-1882. Ottawa 1882.

14. Pour référence, voir note 8 , supra.

15. O'Sullivan, Henry, Second Report of Progress of Exploration in the country between Lake St. John and James Bay (Department of Colonization and Mines, Quebec), 79 p., 1901. (Voir P. 41) Réédité en 1926 sous le titre de "Report of Progress of exploration..." "

16. Michadx, F.A., Histoire des arbres forestiers de l'Amérique Septentrionale, Paris, 3 vol., 1813. (Voir Vol. 1, p. 49). 
reports should cease. "Il est bon de souligner d'abord que le passage cité par O'Sullivan ne vient pas, contrairement à ce qu'il écrit, du journal de Michaux père, paru à Philadelphie en 1888, mais d'un ouvrage de son fils, paru en 1813, texte qu'il a copié sans doute dans l'étude de Brunet de 1861. En outre, contrairement à ce qu'affirme O'Sullivan, le journal de Michaux n'a pas paru dans le Bulletin of the American Geographical Society, mais dans les Proceedings of the American Philosophical Society. Enfin, O'Sullivan lui-même ne s'est jamais rendu dans le territoire situé à mi-chemin entre le lac des Cygnes et le lac Albanel et qui, aujourd'hui encore, reste inexploré pour toutes fins pratiques. Toutefois, l'ayant survolé à maintes reprises, je puis affirmer que la description de Michaux fils cadre bien avec les faits.

Le frère Marie-Victorin ${ }^{17}$, dans ses Études floristiques sur la région du lac Saint-Jean, paru en 1925, consacre quelques pages au voyage du botaniste français et réédite, entre autres, la partie du journal de 1792 couvrant le trajet de Tadoussac à la rivière Rupert et retour. C'est le premier auteur à mettre expressément en doute l'interprétation littérale du trajet de Michaux. Prenant comme base de comparaison une carte du Père Laure, ${ }^{18}$ il conclut à l'inexactitude de la carte compilée par l'abbé Brunet. ${ }^{19}$ Prenant à la lettre les distances mentionnées par Michaux, - toutes basées sur une impression rapide et amplifiée par le verre grossissant de la fatigue et des difficultés, il trace un arc de cercle à quarante-cinq lieues de l'embouchure de la rivière Mistassini, afin de retrouver le lac des Cygnes du botaniste. S'appuyant sans doute sur une mauvaise carte, il croit que le lac File-Axe ${ }^{20}$ se déverse dans le lac Mistassini, quand il appartient en réalité au bassin hydrographique du lac Saint-Jean. Il est d'avis que le portage du Monte à-peine est à la hauteur des terres. Pourtant

17. Marie-Victorin, F., Études floristiques sur la région du lac St-Jean. Contrib. lab. bot. Univ. Montréal, no 4, 1925. (Voir p. 22-38).

18. L'auteur ne dit pas laquelle des nombreuses variantes de la carte de Laure. Souvent très différentes l'une de l'autre, il faut éviter de baser une opinion sur une seule.

19. Pour référence, voir note 9. Cette carte de Brunet était elle-même inspirée d'une carte de Laure.

20. Ce lac, à mon avis, devrait se nommer lac à la Meule, suivant la coutume des Indiens et des forestiers qui fréquentent ces parages. Il semble que le nom de lac File-axe, qui est officiel, soit apparu pour la première fois dans le rapport d'O'Sullivan. Pour référence, voir note 15 supra. 
Michaux s'est exprimé clairement sur ce point dans le journal du 11 septembre, lorsqu'il écrit que, " le lac des Cygnes [ qui est en amont du Monte-à-peine ] se décharge dans le fleuve Snt Laurent par la riv. Mistassin... " Après avoir affirmé que " le problème de la route de Michaux reste sans solution, " il conclut: " Il nous paraît douteux que Michaux ait constamment suivi la Mistassini proprement dite, comme on l'admet généralement. Son itinéraire s'expliquerait beaucoup plus facilement si l'on admettait qu'à l'endroit où la Mistassini passe des hautes terres aux basses terres et s'élargit subitement dans le canton Beaudet, Michaux a pris à sa gauche et s'est engagé dans la rivière Washimeska. Dans cette hypothèse, la route devient plus courte, et le lac des Cygnes pourrait être où le las innommé du canton Hertle, ou encore mieux le lac File-Axe du canton Gauvin. "Hypothèse qui ne saurait résister à la critique et qui doit son origine à des cartes par trop déficientes, à l'opinion que le Monte-à-pein s est à la hauteur des terres et à la croyance que Michaux (ou plutôt ses guides) choisit nécessairement la route la plus courte. La ligne droite s'impose presque naturellement dans un pays traversé en tous sens par des chemins, mais le coureur des bois doit se plier à d'autres contingences: la fréquence et la difficulté des rapides, l'abondance et l'état des portages, l'expérience personnelle, autant de raisons qui obligent parfois à prendre le trajet le plus long.

Le texte du frère Marie-Victorin est accompagné, en outre, de deux cartes, tracées par un assistant. La première, le "Tracé général de l'itinéraire de Michaux, de Tadoussac à la rivière Rupert ", ne correspond pas à l'opinion émise dans le texte par le frère MarieVictorin. La seconde est intitulee, "Reproduction d'une partie de la Carte du domaine du Roy en Canada dressée par le P. Laure, miss. j. et dédiée en 1731 à Monseigneur le Dauphin. " Elle reproduit effectivement un secteur d'une carte de Laure, mais le tracé n'est pas fidèle et la place des noms de localités diffère parfois considérablement de l'original. Cette carte donc, n'est pas une reproduction, mais une interprétation.

Dans l'Histoire du Saguenay, qui ${ }^{2}{ }^{1}$ s'inspire sur ce point de l'étude du F. Marie-Victorin, on affirme que Michaux a remonté la rivière Ouiasimeska.

21. L'histoire du Saguenay, depuis l'origine jusqu'à 1870. Rédigée en collaboration. Publication de la Société historique du Saguenay, no 3, 1938. (Voir p. 142-143) 
Le P. Arthème Dutilly, o.m.i., et l'abbé Ernest Lepage, dans une communication sur l'itinéraire de Michaux au congrès de l'ACFAS, en 1943, concluaient: " une meilleure connaissance de la géographie de la région, permet de croire qu'André Michaux n'a pas remonté la rivière Mistassini jusqu'à sa source et traversé le lac Mistassini dans le sens de la largeur pour atteindre la rivière Rupert, mais qu'il a plutồt abandonné la rivière Mistassini au niveau du lac des Cygnes et atteint l'extrémité sud du lac Mistassini par la décharge du lac Waconichi. Il a dû suivre ensuite la rive ouest du grand lac jusqu'à la rivière Rupert. " Le résume publié ${ }^{22}$, ayant dû être écourté, avait été amputé de cette conclusion. En 1945, les auteurs reprennent la même hypothèse ${ }^{23}$ : " The route Michaux followed would be more probably this: he ascended the Mistassini river as far as the latitude of Swan Lake; then, he left the river and, portaging north-west from small river to small river and passed the chain of lakes, he reached the southern extremity of lake Mistassini and followed the south-west coast of the lake as far as Rupert River. " Hypothèse qui rejoint sensiblement celle de Low.

Les travaux des auteurs susnommés, sans être d'accord, sont néanmoins à peu près unanimes pour soutenir que Michaux s'est trompé. Opinion trop hâtive, qui conduit à une interprétation erronée des faits.

Les fausses interprétations de l'itinéraire de Michaux naissent de la croyance erronée qu'il n'existe qu'une route normale pour se rendre au lac Mistassini; erreur amplifiée par la consultation inévitable de cartes imparfaites. Quiconque sait les exigences de la cartographie ne peut ignorer que l'arpentage sommaire d'un pays comme le Québec, requiert un bon siècle, à condition au surplus de pouvoir compter sur l'abondance des techniciens. De grandes régions, laissées de côté par le cartographe, sont parcourues par de rares voyageurs, - blancs ou indiens, - qui se transmettent oralement leurs connaissances géographiques depuis des générations. Les cartes de ces secteurs, basées sur des récits et des croquis rudimentaires faits souvent après

22. Dutilly, P. Arthème, et Lepage, l'abbé Ernest, La ivière Rupert et le lac Mistassini... l'itinéraire du voyage d'André Michaux en 1792. "Annales de l'ACFAS, 10:95. 1944.

23. Lepage. Abbé Ernest, et Dutilly, P. Arthème. a Retracing the route of Michaux's Hudson's Bay Journey of 1792 ". Revue de l'Universile d'Ottawa, janviermars 1945. Aussi tirage à part de $15 \mathrm{p}$. 
coup, - sont forcément des œuvres de ouï-dire. Les rejeter en bloc, serait se priver d'excellents renseignements, mais l'étranger au terr-to re ne les utilisera qu'avec grande circonspection. Les cartes, même parfaites, exigent une grande expérience de la vie des bois pour servir à la reconstitution d'un itinéraire de voyage. Les portages sont faciles lorsqu'on les effectue en chambre close, les épaules allèges, confortablement assis à une table. En un instant, la pointe du crayon franchit des distances incroyables! Sur le papier, absentes les collines abruptes, les tourbières où l'on s'embourbe, les épinettes trop drues, les charges de mulet, les suées âcres.

Pour aborder convenablement le problème de l'itinéraire de Michaux, il importe de bien étudier tous les trajets qui conduisent au lac Mistassini, cet exposé dût-il contenir des longueurs. Ce sera d'ailleurs une base pour la discussion des voyages du Père Albanel, de Louis Jolliet et de tous ceux qui s'acheminèrent jadis vers la mer du Ncrd.

Au moment où l'avion est venu interrompre les portages entre le lac Saint-Jean et le lac Mistassini, une rivière surtout conduisait les voyageurs au grand lac; mais ce serait une erreur grave que de se laisser impressionner par ce fait. Cette route, tout simplement, est plus pratique pour rallier le poste de Mistassini, maintenant situé à l'extrémité sud-ouest du lac. Le choix dépend aussi, dans une grande mesure, des guides employés. Chacun préfère son trajet familier.

Les renseignements sur les divers itinéraires sont tirés des récits des voyageurs et des cartes géographiques, consultés à la lueur de l'expérience personnelle et des témoignages des habitants occasionnels de la région située entre le lac Saint-Jean et le lac Mistassini, Montagnais de la Pointe-bleue et Mistassini, que les anglophones nomment Cris, à cause de leur parenté linguistique avec les Cris de l'ouest.

Les récits d'Albanel et de Jolliet ne sont d'aucun secours. Ces précurseurs ne cherchaient pas tant à explorer la région entre le lac Saint-Jean et le lac Mistassini, qu'à accomplir à la baie James leurs missions politique, économique et évangélique. Les explorateurs récents contribueront davantage. James Richardson ${ }^{24}$ se rend en 1870 au lac

24. Richardson, James, Report on the country north of lake St. John, by Mr. James Richardson, addressed to A.R.C. Selwyn, Esq., F.G.S., director of the geological Survey of Canada. p. 283-308 du Report of Progress for 1870-71. Geological Survey of Canada, Ottawa, 1872. 


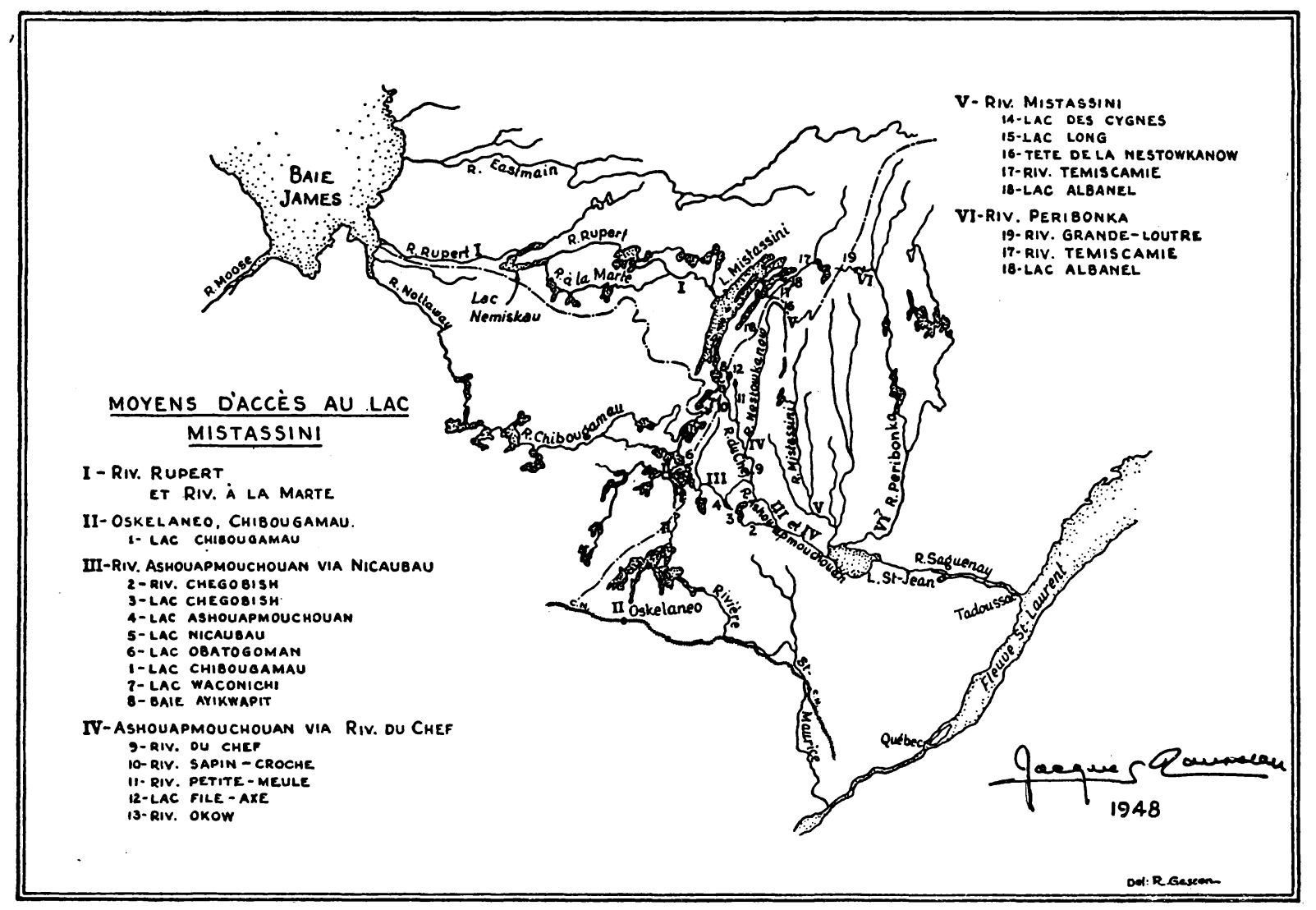

\section{RUPERT}

RT Riv. A La MaRTE

TIN - MEULE 
Mistassini en passant par la rivière Ashouapmouchouan, le lac Nicaubau et le lac Chibougamau. Walter McOuat ${ }^{25}$, pour continuer les travaux de Richardson, atteint le lac Mistassini en 1871 en montant la rivière Mistassini jusqu'à une distance de 105 milles, puis en gagnant la rivière du Chef à l'ouest, pour arriver finalement au grand lac par la baje Cabistachouan (nommée Opitouk par les Indiens). Cette traversée de la rivière Mistassini à la rivière du Chef n'est pas habituelle et McOuat l'a entreprise pour rechercher des terrains cuprifères analogues à ceux qu'avait repérés plus à l'est Richardson en 1870 . James Galbraith ${ }^{26}$, en 1881, va du lac Supérieur à la baie James et de là au lac Mistassini et à Tadoussac, passant par les lacs Waconichi, Chibougamau et Nicaubau. En 1884, John Bignell ${ }^{2} 7$ et Albert Peter Low ${ }^{28}$, après une gigantesque partie de cache-cache à travers la rivière Bersimis, le lac Manouan, la Péribonka et la rivière Témiscamie, atteignent le lac Mistassini au plus fort de la discorde ${ }^{2}{ }^{9}$. La Péribonka, mais non la Bersimis, était une voie normale. Bignell tenait à ce trajet ab̌errant pour relier ses arpentages antérieurs. En 1889, Loudon et MacDonald, pour élucider le " mystère du lac Mistassini " ${ }^{30}$, qui pourtant n'avait rien de mystérieux, l'atteignent par l'Ashouapmouchouan, les lacs Nicaubau, Obatogoman, Chibougamau et Waconichi. O'Sullivan ${ }^{31}$, qui a exploré longuement le territoire entre

25. MCOUAT, Walter, Report on exploration of country between lake St. John and lake Mistassini, by Mr Walter McOuat, addressed to Alfred R.C. Selwyn, Esq., F.G.S., director of the Geological Survey of Canada. Report of progress for 1871-72, Montreal, 1872.

26. Galbraith, John, Canoe-trip from lake Superior to Hudson bay and return via lake Mistassini and the Saguenay river to Tadoussac. Read before the Society on the 8th January 1885. Bull. Soc. de Géog. Québec, 1 (No 4): 1-12. 1885.

27. Bignell, John, Rapport de l'expédition de Mistassini. Appendice No 38 de: Rapport du Commissaire des terres de la couronne de la province de Québec pour les douze mois expirés le 30 juin 1885. Québec, 1886.

28. Low, A.P., Report on the Mistassini expedition 1884-85.55p. Part D, Annual Report, 1885, Geological and Natural History Survey of Canada, Montreal 1885.

29. Sur la malencontreuse odyssée Bignell-Low, voir Rodssead, Jacques, Bataille de sextants autour du lac Mistassini. Action Universitaire, 14e année: 99-116. (Janv.) 1948.

30. New York Herald, 1889. Alphonse Gagnon en a publié unie traduction sous le titre de "Le mystère du lac Mistassini dévoilé ". Voir GAGNon, Etudes archéologiques et variétés, Librairie Beauchemin, Montréal 1913.

31. O'Sullivan, Henry, Second report of progress of exploration in the country between lake St John and James bay, made under instructions from the Department of Colonization and Mines, Québec. 79 p., 2 cartes, Québec 1901. 
le lac Saint-Jean et la baie James, décrit minutieusement les deux routes de l'Ashouapmouchouan, l'une par les lacs Nicaubau, Chibougamau et Waconichi, l'autre par la rivière du Chef et le lac File-axe. Frédéric Pauli ${ }^{32}$, en 1907, atteint l'extrémité sud-ouest du lac Mistassini en passant par l'Ashouapmouchouan et le lac Chibougamau.

Ma fréquentation de la région de Mistassini pendant cinq ans, de 1944 à 1948, m'a mis en mesure d'explorer presque entièrement le grand lac, le lac Albanel et la Témiscamie inférieure. Plus précieuses encore les longues conversations avec les Indiens du territoire: le Mistassin Joseph Metawishish a parcouru en tous sens la région, Simon Matabé, revenu à la vie sauvage après vingt-cinq ans d'essai de la vie des villes, connaît les moindres méandres de l'Ashouapmouchouan, Jimmy Bosum, un ancien Mistassin établi à la Pointe-bleue, chasse dans le voisinage du lac Albanel, voyageant toujours par la rivière Mistassini.

Les principales cartes qui peuvent nous être de quelque utilité, sont les suivantes:

A - Map of Labrador peninsula, south-west sheet. $25 \mathrm{mi}$. au pouce. Compilée par D.I.V. Eaton. Pour accompagner le rapport d'A.P. Low, Report on exploration in the Labrador peninsula... in 1892-93-94-95. Ottawa 1896.

$\mathrm{B}$ - Plan d'arpentage de la rivière Mistassini et de la rivière Samaqua, par Fernand Fafard, Ministère des Terres et Forêts, Québec, 1927. 40 chaînes au pouce.

C - Plans faits sous la direction de Henri Bélanger, arpenteur, et remis au Ministère des Terres et Forêts, Québec, en 1929. Ces plans comprennent: le las Mistassini, le lac Albanel, le territoire entre le lac Chibougamau et l'extrémité sud-ouest du lac Mistassini. 40 chaînes au pouce.

Methes

D - Côte Nord du St-Laurent. Ministère des Terres et Forêts. Québec, 1934. $6 \mathrm{mi}$. au pouce. (Le feuillet ouest couvre une partie du territoire qui nous intéresse).

E - Plan de la rivière Mistassini, par Robert Dorval, Ministère des Terres et Forêts, 1939. 40 chaînes au pouce.

32. PadLI, Frederick K.G., Chibogamoo, 190\%. A record of a trip through Canada's wilderness to lake Chibogamoo and the great lake Mistassini in the summer of 1906 by Fred. K.G. Pauli. Privately printed, New York, 1907. 61 p. et 26 fig. 
F - Territoires d'Abitibi et de Mistassini. Ministère des Terres et Forêts, Québec 1941. 6 mi. au pouce. (Deux feuillets).

G-Mistassini. Air Navigation edition. Hydrographic Map Service, Department of Mines and Resources, Ottawa. 1943. $8 \mathrm{mi}$. au pouce.

$\mathrm{H}$ - Carte régionale, no 3, feuillet ouest (secteur du lac SaintJean), Ministère des Terres et Forêts, Québec 1943. 3 mi. au pouce.

I - Québec, Ministère des Terres et Forêts, 1946. 16 mi. au pouce.

$J$ - Routes from Lake St. John to Lake Mistassini. P. 167 de Delanglez, Life and voyages of Louis Jolliet $^{3}{ }^{3}$.

K - Région du lac Mistassini, Jacques Rousseau 1947. P. 253 de Delanglez, Life and voyages of Louis Jolliet. Cette carte accompagne les notes de Jacques Rousseau sur le voyage de Louis Jolliet à la baie d'Hudson.

L - Carte de Louis Jolliet, 1679, citée plus loin (Voir note 38).

M - Les cartes du Père Laure, dont je connais cinq versions: a) Carte du Domaine en Canada dediee a monseigneur le dauphin... P. Laure, J. a chekoutimi le 23e d'aout 1731 ... " Cette carte des Mistassins depuis Piegouagami jusqu'a Nemiskau doit paroitre d'autant plus fidelle que je l'ai faite avec l'aide des Srs Dorval - des Groisiliers petits Neveux du Sr Medatchouard, lesquels ont hyverné l'un 2 et l'autre 3 ans avec succes aux grands Mistassins pour M.M. de la Compagnie d'occident "). Dimensions du ms. original, $82 \mathrm{~cm} . \times 56 \mathrm{~cm}$. Paris, Bibliothèque du Service hydrographique de la Marine, ms. 4044-B, no 17. Reproduction, Archives canadiennes, Ottawa. - b) " Carte du domaine du Roy en Canada Dressée par le Pere Laure Missionnaire J. 1731 Augmentée de nouveau reveüe et corrigée avec grand soin. en attendant un exemplaire complet l'automne 1732 ". Tracé de le Moyne. Dimensions du ms. original $46 \mathrm{~cm}$. X $34 \mathrm{~cm}$. Paris, Bibliothèque du Service hydrographique de la Marine, ms. 4044-B, no 18. Reproduction, Archives canadiennes, Ottawa. - c) "Carte du domaine du Roy en Canada Dressée par le P. Laure Miss. Jes.

33. Cette carte est basée sur la carte d'Eaton (carte décrite aans le texte) et les notes de Jacques Rousseau sur le voyage de Louis Jolliet. (Pour référence, voir note 4, supra). La carte préparée sous la direction du père Delanglez était de 25 milles au pouce, ainsi qu'on peut le voir à la p. 167 de l'étude du père Delanglez, mais par suite de la réduction photographique, l'échelle est maintenant d'environ 90 milles au pouce. 
1731 Augmentée de nouveau reveüe et corrigée avec grand soin en attendant un exemplajre complet l'automne 1732'). J'ignore où se trouve l'original. Copie aux Archives canadiennes, Ottawa. - d) ( Carte du domaine du Roy en Canada dressée par le P. Laure miss. J. et dédiée en 1731 à Monseigneur le Dauphin. Augmentée de nouveau revue et corrigée avec grand soin par le même en attendant un exemplaire complet 1732. Guyot f. 1733. " Carte en couleurs reproduite dans la Rochemonteix, "Les Jésuites en Nouvelle-France ) (64 cm. X 45.5 cm.) - e) " Carte du domaine du Roy en Canada Dediée à Monseigneur Le Dauphin Parle Pere Laure Jésuite missionnaire de ces endroits mise au net et corrigée sur de bons mémoires par le Sr Guyot Président du grenier à Sel de Versailles 1733. Original se trouvant à la Bibliothèque du Dépot des cartes et plans de la Marine à Paris. Copie $(64 \mathrm{~cm}$. X $34 \mathrm{~cm}$.) dans le "dossier de la cause de Mingan ". (Le titre exact est: "In the privy council. Maps and charts fyled as exhibits in the case Re Labrador Company, appellant, and the Queen, respondent, and E. Contra. " 1891).

On peut ramener à six les différentes routes connues des Indiens et susceptibles de conduire les Blancs au lac Mistassini. De celles-ci seules les quatre dernières intéressent le voyageur du lac Saint-Jean. D'autre part, sont omis ici les portages qui conduisent chaque année des Indiens du lac Nichikoun au lac Mistassini ou de la Eastmain au grand lac, puisqu'ils ne sont pas des intermédiaires pour conduire les Blancs venus de la vallée du Saint-Laurent.

1. Rivière Rupert. Voie normale pour passer de la baje James au lac Mistassini. La Rupert amenait chaque année les Mistassins à la baje James pour y transporter les fourrures du poste et en rapporter ensuite les provisions d'hiver. Cette route abandonnée pour fins économiques depuis quelques années, présentait un double choix sur une partie du parcours: la Rupert proprement dite et la rivière à la Martre. Quand les Français, comme Albanel, Louis Jolliet et André Michaux se rendaient à la baie d'Hudson par le lac Saint-Jean, la rivière Rupert était la seule voie normale au delà du lac Mistassini ${ }^{3}$.

2. Oskelaneo-Chibougamau-Mistassini. Pour l'ancien voyageur

34. Pour une description du trajet cle la rivière Rupert, voir Low, A.P., Report on the Mistassini expedition 1884-85. 55 p. Part D. Annual Report, 1885. Geological and Natural History Survey of Canada, Montreal 1885. 
venant des Trois-Rivières, c'était une voie normale ${ }^{35}$. Route " commerciale " des Mistassins, depuis qu'ils ont abandonné la Rupert, des représentants de chaque famille la parcourent l'été pour aller chercher à Oskelaneo une partie des provisions d'hiver. Voie pratiquement vouée à l'abandon. Quand le chemin du lac Chibougamau sera terminé (dans un an peut-être), ce dernier lac deviendra le terminus commercial des Mistassins.

3. Ashouapmouchouan via Nicaubau. L'itinéraire est le suivant: lac Saint-Jean, rivière Ashouapmouchouan, rivière Chegobish, lac Chegobish, la: Ashouapmouchouan, rivière Nicaubau, lac Nicaubau, lac Obatogoman, lac Chibougamau, lac Waconichi, puis le lac Mistassini qu'on aborde par la baie Ayikwapit (ou baie du Poste, longue de treize milles). A l'embouch rre de la baje du Poste, à la Petite Passe, se trouve le magasin de la compagnie de la baie d'Hudson. Vient ensuite la baie Abatagouche, (longue de quinze milles). Après avoir franchi la Grande Passe, on atteint la partie principale du lac en doublant la pointe cinq milles plus loin. C'est ici que traversent les canots se rendant à la Rupert. Parvenus sur la rive nord-ouest du lac, ils la longent pendant douze milles, dans la direction nord-est, avant de pénétrer dans la baie Duhamel du Monceau où l'on fait neuf milles avant de prendre, au-delà de l'île de la Grosse Roche ${ }^{36}$, le très court portage qui accède à la Rupert et permet d'éviter un détour de quinze milles. Cette route est très populaire aujourd'hui ${ }^{3}$.

4. Ashouapmouchouan via rivière du Chef. Le trajet est le suivant: rivière Ashouapmouchouan, rivière du Chef, rivière Sapin-croche ou

35. Cette route a fait l'objet d'une description détaillée. Voir: "Canoe trip Oskelaneo to lake Chibougamau and lake Mistassini "). (208 miles). Department of the Interior, National Development Bureau, Canoe route No 8, [ Ottawa] 1937. Itinéraire polycopié accompagné d'un plan couvrant tout le trajet, à l'échelle de 8 milles au pouce. Voir aussi: "Route de Chibougamau de la gare d'Escalana sur le chemin de fer Canadien National au lac Chobougamau. "Département des Terres et Forêts, Service des Arpentages, Québec 1929. Carte en deux feuillets, sous couverture, à l'échelle de trois milles au pouce, préparée par Charles Savary, cartographe. Cette carte ne couvre pas le trajet du lac Chibougamau au lac Mistassini.

36. Grosse roche se dit mista assini dans le dialecte local. Ce gros bloc erratique a donné son nom au lac Mistassini.

37. Route décrite (itinéraire et plan) dans la publication polycopiée suivante: "Canoe trip from lake St-John to lake Mistassini (238 miles) ). Natural Resource Intelligence service, Departmerit of the Interior, [ Ottawa] 1937. Échelle de la carte 7.89 milles au pouce. 
rivière Petite-meule, au choix, le lac 'File-axe (lac à la Meule), la rivière Okow, baie Ayikwapit, ba:e Abatagouche, lac Mistassini proprement dit. A la fin, il peut y avoir une légère variante. Si l'on ne veut pas se rendre à l'emplacement du poste actuel, on pourrait passer du lac à la Meule à la baie Opitouk (généralement désigné sur les cartes par le nom de Cabistachouan), par l'extrémité ouest du canton McOuat. La baie Opitouk débouche dans la baie Abatagouche. La route de la rivière du Chef (mais avec arrivée dans la baie du Poste) est très populaire aujourd'hui.

5. Rivière Mistassini. Remonter la rivière Mistassini jusqu'à la latitude $49^{\circ} 50^{\prime}$ environ; passer par le portage de Monte-à peine au lac des Cygnes (que l'on désigne encore sous le nom de Swan Lake actuellement, même sur les cartes officielles de la province de Québec, bien que le nom lac des Cygnes date du régime français ainsi qu'en font foi les cartes du Père Laure) $3^{37^{a}}$ puis, lac des Iles et lac Long et les nombreux lacs et portages qui les relient; de la tête du lac long, par un court portage, on passe à la source d'une rivière qui coule vers le nord. Les anciens voyageurs, comme Michaux et Jolliet, croyaient que cette rivière se déversait dans le lac Mistassini, par l'intermédiaire du lac Albanel. La carte de Jolliet de 1679 la représente ainsi ${ }^{3}$. Contrairement à l'opinion de Jolliet et Michaux, il s'agit de la tête de la Nestowkanow. D'après les témoignages des Indiens Jimmy Bosum et Joseph Raphaël, la Nestowkanow se dirige de la source vers le nord sur une distance de cinquante milles, puis se replie brusquement vers le sud pour se déverser dans le lac Saint-Jean par l'Ashouapmouchouan. Entre le coude de la Nestowkanow et l'embouchure de la Témiscamie, il n'y a qu'un court portage (la ligne de partage des eaux se trouvant

37A. Depuis la rédaction, j'ai reçu une communication de M. Isaïe Nantais,secrétaire de la Commission de Géographie de Québec, me faisant part que ce lac portera désormais l'ancien nom de lac des Cygnes.

38. Louis Jolliet, 1679. " Cette carte montre le chemin que Louis Jolliet a fait depuis Tadoussac iúsqu'à la mer du Nord, dans la baye d'Hudson, et la vraye scituation de la baye et du d'estroit, ce qui est marqué de rouge est le chemin par ou il a été. Fait à Quebec, le 8 me noembre 1679. L. Jolliet. ") Copie ms mal conservée, sur papier, $77 \times 52 \mathrm{~cm}$. Archives canadiennes, Ottawa. Original conservé au Dépôt des Cartes et Plans de la Marine (i.e. Archives du Service hydrographique), Paris, document 123-8-1. Sur la copie d'Ottawa, il n'y a pas de tracé en rouge. Il existe au moins trois variantes de cette carte de 1679. Le père Delanglez les décrira dans l'édition française de la "Vie de Louis Jolliet ». Voir aussi note 4 supra. 
beaucoup plus au nord que ne'l'indiquent les cartes). En s'engageant dans le portage, les voyageurs étrangers croyajent éviter des rapides situés près de l'embouchure de la rivière. Du lac Albanel on passait ensuite au lac Mistassini par le portage d'Opitchouan ou celui des Pieds-bralants (Kapochepouchekochitéchinenaneoutch pour les Mistassins). Cette route, qui semble avoir été populaire, n'est plus fréquentée aujourd'hui, sauf par les quelques Indiens qui ont leur territoire de chasse dans ces parages ${ }^{3}$.

6. Rivière Péribonka. Au point de départ, deux variantes: a) rivière Saguenay, lac Kénogami, lac Saint-Jean et rivière Péribonka; b) rivière Saguenay, rivière Shipshaw et portages vers la Péribonka, entre le $49^{\circ}$ et le $49^{\circ} 30^{\prime}$ lat. N. Puis, dans un cas comme dans l'autre, montée de la Péribonka jusqu'à $50^{\circ} 25^{\prime}$, rivière Saint-Onge, lac Onistagan (on peut passer aussi directement de la Péribonka au lac Onistagan), de nouveau Péribonka jusqu'à $51^{\circ} 10^{\prime}$ environ lat. N.; puis vers l'ouest, rivière Grande-loutre (en réalité une série de lacs reliés l'un à l'autre), grand lac Témiscamie, rivière Témiscamie jusqu'à son embouchure, lac Albanel, portage d'Opitchouan ou celui des Piedsbrulants, lac Mistassini. Pour se rendre à la Rupert (et il en est de même avec l'itinéraire de la rivière Mistassini), le portage d'Opitchouan est tout indiqué. Rendu dans les eaux du grand lac, on se dirige vers le nord, pour doubler l'île Tchapahipan par le Grand Percé, puis de là on passe à la rive nord-ouest du lac qu'on longe jusqu'à la Rupert. Chemin peu fréquenté, Jolliet est néanmoins passé par là en 1679.

Un autre point qu'il importe également d'envisager est l'emplacement des postes de traite du lac Mistassini lors de la visite de Michaux. Il n'en mentionne aucun; pourtant, s'il en avait rencontré, son journal circonstancié en garderait trace: quand on a couru la solitude pendant deux semaines, un poste aurait semblé une oasis.

39. Lorsque j'ai remis au père Delanglez un mémoire sur le trajet de Louis Jolliet au Lac Mistassini, et qu'il publia intégralement en appendice dans sa remarquable biographie (voir note 4 supra), j'éloignais l'hypothèse d'un voyage de Jolliet par la rivière Mistassini à cause d'omissions et surtout d'une erreur de cartographie imputable à la méconnaissance de la longitude. J'ignorais alors que les anciens n'avaient aucun moyen pratique pour déterminer la longitude. Toutefois, - ce qui aurait dû me mettre en garde contre le rejet de l'hypothèse, - je reconnaissais que le fait d'avoir tracé le cours d'une " rivière se jetant vers le nord " était conforme aux connaissances de l'époque. J'aurais dâ même considérer ce fait comme un indice que Jolliet était passé par là. 
Sur l'histoire des postes, Voorhis ${ }^{40}$ sera notre meilleur guide. Le fort Mistassini, de la chaîne des postes du Roy, aurait été fondé en 1673 sur la presqu'île Eliquabit (aujourd'hui Ayikwapit, dans la baie du même nom à l'extrémité sud-ouest du lac Mistassini). Si Michaux avait voyagé par l'un des deux trajets de l'Ashouapmouchouan, et avait abordé le lac Mistassini par l'extrémité sud-ouest, il serait sûrement arrêté au poste suivant une coutume indéfectible. Ce poste fut acquis en 1802 par la Compagnie du Nord-ouest. La compagnie de la baie d'Hudson, installée en 1787 sur la Eastmain, à Neoskweskau, transporta son poste en 1800 près de la décharge du lac Mistassini, puis en 1835 à l'endroit actuel. Le poste de la compagnie du NordOuest avait cessé de fonctionner lors de l'union des compagnies en 1821.

\section{SourCES ORIGINALES DE RENSEIGNEMENTS}

SUR Les VOYAges DE MichauX

En 1824, François-André Michaux, fils d'André et auteur de l'Histoire des arbres forestiers de l'Amérique septentrionale, donnait à l'American philosophical Society, de Philadelphie, le journal de voyage de son père en Amérique pour les années écoulées entre 1787 et 1796 , les cahiers de 1785 et 1786 ayant été perdus dans un naufrage. Le journal de 1792, qui relate le voyage au lac Mistassini, est intact. François-André se porte garant de l'authenticité du manuscrit: " $\mathbf{S i}$ ces journaux que j'offre à la Société philosophique de Philadelphie (à laquelle j'ai l'honneur d'appartenir) ne renferment rien qui soit utile aux Botanistes américains à venir, au moins ils pourront être assurés d'avoir sous les yeux, les notes tracées par la main d'un homme qui consacra une grande partie de son existence au progrès de la

40. Voorhis, Ernest, Historic forts and trading posts of the French regime and of the English fur trading companies. Travail polycopié, Ministère de l'Intérieur, 1930. Voir aussi [ WhITE, James ], Forts and trading posts in Labrador peninsula and adjoinirg territory. In the privy council: in the matter of the boundary between the Dominion of Canada and the Coiony of Newfoundland in the Labrador peninsula. Between the Dominion of Canada of the one part and the Colony of Newfoundland of the other part. Ottawa, F.A. Acland, 67 p. 1926. Cette partie du factum ne me semble pas avoir été comprise dans le grand dossier de la cause. 
Botanique. ") ${ }^{4} \mathrm{Au}$ paragraphe précédent, le fils nous renseigne sur la façon de procéder de son père pour la rédaction du journal: "Campant presque toujours dans les bois, s'étoit la nuit à la lueur du feu que mon père écrivoit les remarques qu'il avoit faites dans le jour. " Sans aucun doute, l'auteur épuisé par une journée de travail pouvait oubler à la brunante des faits qu'il n'aurait pas omis en écrivant au fur et à mesure des événements, mais le recul n'était pas assez considérable pour estomper les faits.

Les collections du Jardin botanique comptent une copie photographique du journal manuscrit de 1792; mais nous pourrions aussi bien nous en remettre à la publication de Sargent. ${ }^{4}$ Si la trans sription n'est pas parfaite, les variantes en général n'ont pas une importance capitale.

Les spécimens botaniques recueillis par André Michaux durant le voyage sont encore conservés au Museum d'histoire naturelle à Paris. Les étiquettes, de la main du collecteur, portent de précieux renseignements sur l'habitat. Les collections du Jardin botanique de Montréal et de l'Institut botanique de l'Université de Montréal comptent plusieurs photographies de spécimens originaux. D'autre part, un manuscrit de l'abbé Ovide Brunet, ${ }^{4} 3$ ntitulé " Notes sur l'herbier de Michaux au Jardin des Plantes (Paris)" renferme la copie du texte de plusieurs étiquettes. Cependant, quel que soit l'intérêt de ces notes manuscrites, elles n'apportent aucune précision sur l'itinéraire.

La Flore de Michaux, ${ }^{44}$ rédigée par Louis-Claude Richard et publiée en 1803, un an après la mort de son auteur présumé, donne des indications sur la récolte des plantes entre le lac Saint-Jean et le lac Mistassini; mais ces commentaires n'ajoutent rien qui ne soit déjà dans l'herbier et le Journal.

Michaux fils, dans l'Histoire des arbres forestiers, ${ }^{45}$ cite de rares

41. Lettre reproduite par Sargent dans son édition du Journal de Michaux. Pour références, voir note 8 , supra.

42. Pour références, voir note 8 , supra.

43. Archives du Séminaire de Québec. Ces notes manuscrites ont servi à la rédaction de la " Notice sur les plantes de Michaux... " Pour références, voir no 9.

44. Michadx, André, Flora boreali-americana... 2 vol. 330 et 340 p. Première édition, Paris 1803. Deuxième édition, sans changements, Paris 1820.

45. Mrchaux, François-André, Histoire des arbres forestiers de l'Amérique septentrionale, 3 vol., Paris 1813. 
textes manuscrits de son père, non compris dans le Journal, mais aucun n'apporte d'éclaircissement à la présente étude.

Il ne nous reste donc que le Journal même, qu'il faut étudier à la lueur des cartes modernes, aussi imparfaites qu'elles soient, car la cartographie de la région située entre le lac des Cygnes et le lac Albanel est à peine esquissée.

\section{INTERPRÉtation De L'Itinéraire De Michaux}

Le voyage de Michaux au Canada débute le 7 juin 1792 . Le voyageur nous annonce qu'il s'est alors " préparé à partir pour le Canada ". Le 14 il passe à Albany, le 20, au lac Champlain. Après quelques jours d'herborisation, le voilà entré le 28 juin en territoire canadien. Le 29 à Saint-Jean, il est à Montréal le 30 juin. Jusqu'au 30 juillet, herborisation entre Montréal et Québec. Le 30, préparatifs pour le voyage au lac Mistassini et départ de Québec le lendemain. Arrivé à Tadoussac le 5 août, le 10 il est à Chicoutimi et le 12, charge deux canots des provisions dont il aura besoin pour son voyage. S'engageant le lendemain dans la rivière Chicoutimi, le 15 août il campe à la sortie du lac Kénogami.

C'est à partir de ce point que nous suivrons pas à pas Michaux, tentant, au moyen de son journal, d'établir la route parcourue. Chaque journée de voyage fera généralement l'objet de deux paragraphes: le premier, en italique, débutant par la date, résume le trajet de la journée. Des extraits toutefois seront cités littéralement d'après le manuscrit. Ce sont ceux entre guillemets. Dans ce cas, je souligne en notes infrapaginales les variantes de Sargent si elles ont quelque importance. Pour les simples variantes orthographiques, je me contente de citer dans le texte la forme manuscrite exacte. Le paragraphe suivant, en caractères romains, comprendra les commentaires. Certains jours, ils feront défaut.

15 août 1792, " Avons fini la journée en passant le lac Senogamie. qui a sept lieues de long et entres ${ }^{46}$ un et deux Miles de largen.

Le lac Kénogami mesure 21 milles de long, d'après Rouillard, 47

46. Dans Sargent, autres, au lieu de entres.

47. Rouillard, Eugène, Dictionnaire des rivières et des lacs de la province de Québec. Département des Terres et Forêts, Québec 1914, 432 p. 


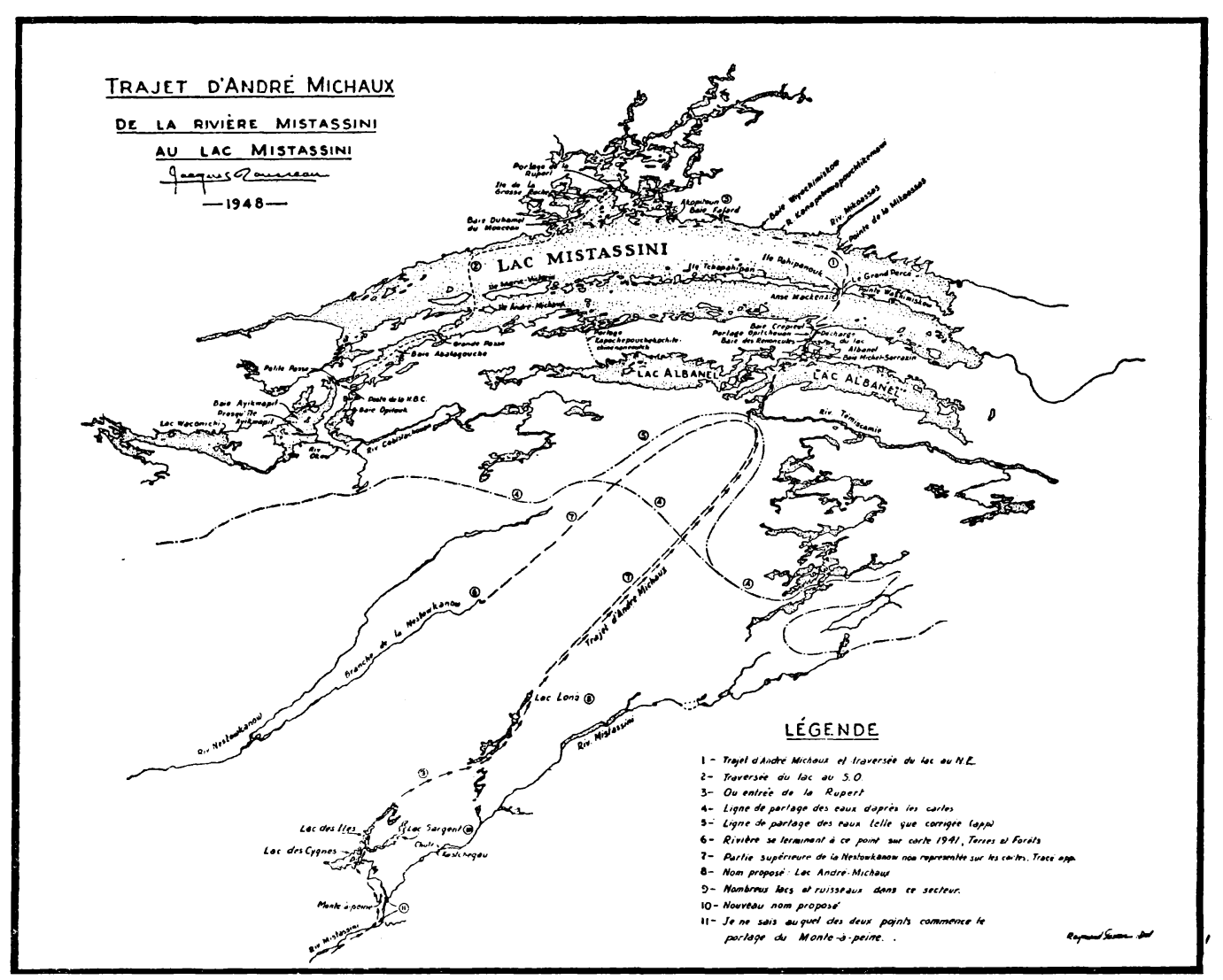

菅

曷

INTERPRÉTATION DE L'ItinéraIre DE MichauX. 
quoique un relevé libéral sur la carte régionale no 3, 1943 (précédemment citée) ne donne que 18 milles.

16 août. Deux portages entre le lac Senogamie et une rivière coulant vers le lac Saint-Jean. Michaux affirme faire trois liene à l'heure sur cette rivière. Après un trajet de 10 lienues [ depuis le lac Senogamie] Michaux campe à l'embouchure, où des Artemisia croissent sur des collines de sable.

La rivière coulant vers le lac Saint-Jean est la Belle-rivière. Bien que le niveau du lac ait été haussé, il y a encore à l'embouchure de grandes dunes de sable où crô̂t l'Artemisia canadensis. C'est de là que vient le type de l'espèce. Une vitesse de neuf milles à l'heure en canot semble extraordinaire. Cela se conçoit pour les plus forts courants et sur de courts trajets. Six à sept milles à l'heure représentent déjà une grande vitesse moyenne. Du lac Kénogami à l'embouchure de la Belle-rivière, il y a au maximum 18 milles. L'exagération vient de deux facteurs: le portage, qui paraît plus long qu'en réalité, à cause des difficultés, et la vitesse du courant qui semble d'autant plus forte qu'on est près de l'eau. A voir filer les cailloux sous le canot, on a réellement l'impression d'une vitesse extraordinaire.

17 août. Le vent contraire empêche d'entrer dans le lac le matin. L'après-midi, ramèrent quatre heures, à un quart de lieue du bord, et cependant les canots touchaient fréquemment au fond.

Dans ces conditions, il est peu probable qu'ils aient fait plus de cinq milles. Un bon lieu de campement qui se présentait tout naturellement est l'embouchure de la rivière Koushpaiganish, emplacement actuel du village de Saint-Jérôme.

18 août. "Nous arrivames vers les 4 heures apres midy au Poste établi pour la Comp.e po. la traite des fourrures av. les Sauvages des Lac des Cygnes et Lac Mistassin. Ce Poste est occupé par deux comis Canadien Mrs Panet freres. ")

On peut se demander ici s'il s'agit du poste de Métabetchouan, dont il reste encore la poudrière à l'embouchure de la rivière Métabetchouan, à Desbiens, ou bien du poste de la Pointe-bleue. Il y a en effet une dizaine de milles seulement entre l'embouchure de la Bellerivière et celle de la Métabetchouan, et de 28 à 32 milles de l'embouchure de la Belle-rivière à la Pointe-bleue. Cette course semblerait plus normale pour deux jours; mais outre que Michaux n'a voyagé 
que quatre heures le 17 , - et dans les plus mauvaises conditions, son journal ne donne aucune idée du trajet fait le lendemain. Un point sûr, c'est qu'un voyageur ne pouvait passer au poste sans y séjourner une nuit. Si l'on avait campé à quatre ou cinq milles du poste le 17, le départ ne pouvait presser le lendemain. D'autre part, nous savons par James McKenzie ${ }^{48}$ que le poste en 1808 se trouvait à " quatre lieues " de l'embouchure de la Belle-rivière. C'est là que le situait le père Laure sur ses cartes, en 1731. C'est également là que Bouchette le trouve en $1828 .^{49}$ Le déménagement à la Pointe-bleue est postérieur à $1864^{50}$.

19 août. "Une brume épaisse nous empêcha de traverser le lac po. entref dans la riv. ditte Mistassin. )

2,1 août. Après deux jours passés au poste à cause de la brume puis de la tempête, " parti du milieu du Lac St Jean ou est établi le Magasin des Marchandises po. la traite av. les Sauvages. C'est le dernier Poste dans ces lieux sauvages. Il étoit neuf heures du Matin et.nous entrames dans la Rivierre ditte Mistassin a 2 heures apres midy. ${ }^{51}$ Nous avons voyagé en Canots dans cette Riv. jusqua huit heures du Soir. Lembouchure dans le lac est tres peu profonde et pendant cinq à six lieues en remontant on voit des bancs de sable mouvans qui ont plus dune demie lieue de long. ")

En longeant sommairement la rive ouest du lac, le trajet, du poste à l'entrée de la rivière, serait d'environ 30 milles, parcourus en 5 heures, ce qui est trop. Le journal du 19 août nous apprend que Michaux devait (" traverser " le lac. Le trajet en ligne directe du poste à l'embouchure de la Mistassini est d'environ 24 milles. Cette vitesse se justifie. Quand les Indiens traversent de grandes nappes d'eau, comme j'ai pu l'observer au lac Mistassini, ils se précipitent, ne prenant aucun repos, ne regardant même pas devant eux pour ne pas éveiller les esprits des tempêtes. - Les bancs de sable du cours inférieur de la Mistassini

48. McKenzIE, James, The King's posts and Journal of a canoe jaunt through the King's domains, 180.8. P. 401-454 de: MAsson, L.-R., Les bourgeois de la Compagnie du Nord-Ouest. Deuxième série, 1890.

49. Rapport des Commissaires pour explorer le Saguenay, 1829. p. 135.

50. White, op. cit. Pour références, voir note 40, supra.

51. Dans SARgent: " dans la Rivierre ditte Mistassin à 2 heures de l'après midy ". Noter dans l'édition Sargent l'abus des accents graves qui font généralement défaut dans le ms. Sargent, notamment, transcrit toujours rivierre par rivièrre. 
sont bien caractéristiques et on en voit encore d'immenses malgré l'exhaussement du niveau du lac.

22 août. "Nous avons continué en remontant la rivierre pendant une heure et ensuite nous avons rencontré un Portage... A l'endroit de ces premiers rapides, la rivierre diminue tout à coup, ayant eu jusqu'ici 3 à 4 Miles de largeur. .)

Dans un ouvrage anonyme du Ministère des Terres et Forêts publié en 1908, ${ }^{5}$ on lit: "La Mistassini, large de 3 milles à son embouchure, est navigable et naviguée par les bateaux à vapeur jusqu'au pied des premières chutes, à environ 24 milles du lac St-Jean et sur tout ce parcours, sa profondeur moyenne est de 5 ou 6 pieds. Au-dessus de ces premières chutes, la largeur de la rivière atteint souvent, excède même en plusieurs endroits, un demi-mille. " La première chute est au $48^{\circ} 55^{\prime}$ lat. N. Sauf à l'embouchure (et encore il faut une traversée oblique) la Mistassini et les autres rivières importantes de la région n'ont guère plus de trois quarts de mille ou un mille de large dans la partie inférieure de leur cours.

22 août, suite. "Nous avons rencontré neuf Rapides et conséquemment nous avons eu neuf Portages a franchir sans quitter cette rivierre ditte Mistassin quoiquelle ne sorte pas des lacs Mistassins. Depuis le depart du Poste sur le lac St. Jeian jusqu'aux Larges Rapide's ou nous arrivames vers les 7 heures du soir la distance est evaluée 18 lieues. Quoique lon considere géngralement les Rapides ou Cascades perpendiculaires comme des Effets de la nature digne de curiorité, lon auroit de la peine a se former une idée de la Majestueuse perspective de celle-ci. Elle est naturellem ${ }^{t}$ crusée, $e^{53}$ en Amphiteatre dans l'enfoncement duquel on ne voit que les Arbres aussi bien que sur les cotés et elle s'élargit $\grave{a}$ sa base d'environ 200 toises sur une profondeur d'env. 250 toises. ${ }^{54}$

Le nom montagnais des Larges Rapides, d'après Joseph Raphael et Jimmy Bosum, est Kissegau. Cette cascade, située vers $49^{\circ} 10^{\prime}$, est à environ 54 milles du lac Saint-Jean, comme l'affirme Michaux

52. Anonyme. Régions de Québec, du lac-Saint-Jean, de Chicoutimi et de la côte nord du Saint-Laurent. - Description des cantons arpentés, explorations de territoires et arpentages des rivières et des lacs. De 1889 à 1908. Mínistère des Terres et Forêts, 280 p., 1908. (Voir p. 52).

53. Dans Sargent: "Elle est naturellement comme un Amphitheatre ".

54. Danls Sargent: "d'environ 250 toises sur une profondeur d'environ 250 toises $)$. 
23 août. Pluie, Pas de voyage.

2i4 août. Deux rapides et consequemment deux portages. "Notre journée peut etre évaluée à 8 lieues. )

Le 16 août, les distances paraissajent un peu exagérées: il en sera de même le 26 et également d'autres fois. Si nous additionnons toutes les distances de la course entre le lac Saint-Jean et le lac Mistassini, nous constaterons que la longueur totale dépasse sensiblement l'objectif. Il faut réduire les données du voyageur de 25 à 33 p.c. Cette déviation ne dcit pas diminuer le crédit à accorder à l'auteur. Quiconque a fréquenté la nature vierge sait que l'impression du voyageur dépasse toujours de façon appréciable la réalité. Les difficultés de la course, les fatigues, sont les causes de cette illusion d'optique. Pour la journée du 24 août, on peut donc évaluer la course à 6 lieues, soit 18 milles.

25 août. Voyage à la perche pour lutter contre le courant. 7 à 8 lieues dans la journée.

Réduire la course à 5 lieues.

25 août. "Nous avons été obligé de faire aller les Canots avec les perches seulement, depuis 7 hourds du Matin jusque vers onze heures. Nous avons rencont é une Cabane de Sauvages et nous y avons diné... Notre Marche fut d'environ six à se.pt lieues. ")

Il semble bien par le contexte que les canots ont navigué quatre heures seulement et parcouru pendant ce temps de six à sept lieues. Or, à la perche, en montant le courant, il est bien difficile de faire plus d'une lieue à l'heure. La course de la journée peut donc se ramener à 4 lieues.

27 août. Rivière extrêmement diminuée de largeur. Courant très rapide. "Notre course peut etre évaluée à 8 lieues. )

Réduire à six lieues. En additionnant tous les trajets ainsi réduits, nous sommes à 63 milles des "Larges Rapides ". Or le portage Monteà-peine, atteint à $2 \mathrm{~h}$. le lendemain, se trouve à environ 60 milles des "Larges Rapides ". Le facteur de réduction des trajets, employé jusqu'ici, se défend donc parfaitement.

28 août. "Les Sauvages ont continué de piquer avec les Perches po. forcer les courans tres rapides et vers deux $h$. apres Midy nous arrivames au Portage Monte a peine. Nous avons été depuis Sh. jusqu'a 7h. du soir pour grimper cette Montagne et pour arriver dans une autre 
petite Rivierre située de lautre coté. J'evalue à 250 ou 300 toises perpendiculaires environ la hauteur de cette montagnc et la Riv. située de lautre coté nest pas de 40 a 50 toises plus bas que le sommet de cette Montagne Monte a peine. Les Sauvages me disent que cette Riv. n'a pas de nom. )

Joseph Raphael, lorsqu'il parle français, emploie le nom Monteà-peine pour ce portage situé vers $49^{\circ} 51^{\prime}$ lat. $\mathrm{N}$. (J'ignore le point de départ précis). Le nom montagnais est Pastchestagan ou Psechtagan que Joseph Raphael traduit par "lorsque l'on prend le portage " et David Cooter, gérant du magasin de la Hudson's Bay Company à la Pointe-bleue, par "turning off main road". Il faut se garder de confondre le Monte-à-peine avec une colline située vers $50^{\circ} 2^{\prime}$ lat. N. et que Jimmy Bosum et Joseph Raphael nomment Kastchegau. Le portage de Monte-à-peine conduit vers le lac des Cygnes et la petite rivière citée semble la décharge de ce lac qui se jette dans la rivière Mistassini vers $49^{\circ} 54^{\prime}$. C'est vers ce point que Michaux se serait engagé dans le portage et, au delà de la colline, il aurait repris le cours d'eau après avoir évité les rapides de la partie inférieure. Il n'est pas impossible aussi que Michaux ait commencé le portage vers $49^{\circ} 48^{\prime}$ lat. N., mais dans ce cas il serait passé par le lac Traverse, long d'environ trois milles et assez important pour être signalé.

29 août. "Nous avons eu quatre portages a passer dans l'intervalle desquels nous avons voyagé sur deux Rivierres qui n'avoient pas plus de 18 pierds de large. " Digues de castors. A \$h. les voyageurs arrivent au lac des Cygnes.

Il y a là deux petits ruisseaux, d'après Jimmy Bosum. Toutefois le plan de la rivière Mistassini en indique trois. Le lac des Cygnes se nomme en montagnais Wabisi wagami, ayant le même sens. Les cartes de Laure portent le nom lac des Cygnes. C'est donc bien à tort que l'on accepte comme officiel le nom Swan Lake. ${ }^{54^{\mathrm{a}}}$

30 août. " Avons navigué dans trois Lacs environné de Montagnes peu élevées qui se communiquent par des issues entre ces collines... Nous avons fait trois portages et nous avons fait env. 3 a 14 lieues a cause de la difficulté a traverser ces desagréables marécages. 》)

Il s'agit soit du trajet depuis le lac des Iles jusqu'à un lac innommé dans le canton Salières, ou bien du trajet par un lac étroit et vague-

54a. La Commission de Géographie, à qui j'ai soumis le cas depuis la rédaction de ce texte, décide de revenir à l'ancien nom, lac des Cygnes. 
ment en forme de croissant apparemment innommé, allant du lac des Cygnes jusqu'au centre du canton Dupré. Pour ce lac en croissant, je propose le nom de lac Sargent, en l'honneur de Charles Sprague Sargent, 1841-1927, anciennement directeur de l'Arnold arboratum de Harvard University, le premier éditeur du journal de Michaux, l'un des botanistes américains qui se sont particulièrement intéressés à la floristique québécoise.

31 août. "Nous avons navigué pendant une heure et nous avons rencontré un Portage. Le froid etoit excessif et le temps couvert depuis 2 jo. et la pluye étoit comme de la neige fondue... Nous avons passé trois Lacs et une riv. d'eau courante. ")

Du 31 août au 2 septembre inclusivement, Michaux voyage entre l'un des lacs au nord du lac des Cygnes (le lac des Iles ou le lac Sargent prénommé) et le lac Long., entre $50^{\circ} 10^{\prime}$ et $50^{\circ} 21^{\prime}$ lat. N., par $72043^{\prime}$ long. W. (carte du Ministère des Terres et Forêts, 1941).

1 septembre, "Le samedy 1er Septembre, la pluye nous empecha de voyaget et un des Sauvages fut malade. La cause me parut etre la transpiration arretée. Il avoit été mouillé de la pluye le jour precedent et il avoit dormi dans sa Couverture qui etoit imbibée d'eau. L'apres midy le temps fut moins obscur et nous avons navigué nonobstant la pluye. Toute la nuit, il y eut Pluye Tonnere et Eclairs. Nous avons fait env. six lieues et Nous avons eu un lac et des rivierres tres étroites ou il ny avoit q. la largeur d'un Canot. )

Sans doute le lac Long, parfois tellement étranglé qu'il n'y a plus qu'un mince filet d'eau et qui peut se ramener à une chaîne de petits lacs, peut-être aussi à un chapelet de lacs glaciaires, ce que les géographes anglais nomment populairement un pater-noster. Lac Long ! l'un des centaines, des milliers de lacs Long de la Province. Ce n'est plus un toponyme, mais un nom commun d'accident géographique désignant un type de lac; de même, lac à la Barbotte, lac des Vases, lac des Sables, lac des Iles. Il faudrait bannir impitoyablement ces toponymes. Pour le lac Long du trajet de Michaux, je propose lac André-Michaux. ${ }^{54^{\mathrm{b}}}$

54b. Depuis la composition de ce texte, j'ai appris du secrétaire de la Commission de Géographie que ce lac Long se nommait lac Cauvet depuis le 15 mars 1945. Le nom de lac André Michaux sera donc donné à un autre lac innommé. 
Q septembre. "Le Dimanche 2 le temps fut tres obscur des le matin et il se resolut en Neiges fondue. Le froid fut moins rude; mais nous avons eu un portage de trois quarts d'une lieue au travers d'une Savanne de Sphagnium ou lon enfonçoit jusqua la moitié des jambes et malgré les ondées de grêle qui continuerent toute la journée nous continuames a voyager, car les Sauvages aussi bien que moi désiroient arriver le plustot possible a Mistassin de peur que les Neiges et les froids ne devinssent plus considerables. Nous avons eu trois lacs a traverser et nous avons fait environ dix lieues. ")

Entre le lac Long et la tête de la Nestowkanow.

3 septembre. "Le [temps] parut bien disposé au moins po. la journée, mais vers 7 heures lair devint nuageux et nous avons eu de la Pluye et alternativ de la Grêle et de la Neige et des Intervalles d'un beau Soleil... A onze heures nous entrames dans une grande rivierre qui coule vers le Nord. Ayant les courants favorable no. avons fait 16-18 lieues. ))

La Nestowkanow, d'après Joseph Raphael et Jimmy Bosum va beaucoup plus loin au nord que ne l'indique la carte et dépasse même d'une quinzaine de milles la ligne de partage des eaux telles que tracée actuellement. A la longitude $73^{\circ} \mathrm{W}$. la ligne de partage des eaux fait une saillie vers le nord jusqu'aux environs de $50^{\circ} 55^{\prime}$ lat. N. La Nestowkanow, qui débuterait dans le voisinage du lac André-Michaux (lac Long), et qui prend peut-être sa source dans ce lac, ce que nous ignorons, - coule d'abord pendant une cinquantaine de milles vers le nord, puis, après avoir frôlé presque le coude de la Témiscamie inférieure, se replie brusquement sur elle-même pour se diriger vers $l$; lac Saint-Jean. La " rivière qui coule vers le nord" ne peut être que la tête de la Nestowkanow. Nous avons vu précédemment que Jolliet croyait cette rivière un affluent du lac Albanel. Au sujet de cette rivière, O'Sullivan écrit ${ }^{5}$ : " The Nestaskano is a magnificent river averaging about 300 feet in width with deep water and steady current as far as we followed it. The Indians say that it drains an immense basin stretching long beyond the parallel of lake Mistassini. " Une course de 45 à 50 milles en canot, en suivant le courant d'une rivière n'a rien de particulièrement extraordinaire, l'expérience me l'a appris.

55. Op. cit., (voir note 31 ), p. 22. 
4 septembre. "Nous av. fait trois fois Portage a cause des courants tres rapides dans les rochers. A 10h. 1/4 entre dans le lac Mistassin... Navigué dans le Lac environ 10 à 12 lieues, campé sur la rive gauche $N$. Ouest a 6 lieues de distance du Lac. )

Ici deux interprétations se présentent. Première hypothèse. Les trois portages sont les suivants: $1^{\text {er }}$ portage, entre le coude de la Nestowkanow, décrit précédemment, et la Témiscamie, au point où celle-ci, à quatre milles de l'embouchure, tourne à angle droit vers le nord-ouest. Michaux ne s'aperçoit pas, comme autrefois Jolliet, qu'il passe d'un système hydrographique à un autre, croyant sans doute que le portage permet simplement d'éviter de forts rapides. 2e portage, dans le lac Albanel, à la décharge du lac, pour passer de la baie MichelSarrazin à la baie des Renoncules, pour éviter le fort courant. J'ai déjà fait ce court portage. Si Michaux n'a pas compté ce portage, le deuxième est un secteur du premier ou du troisième. 3e portage, celui d'Opitchouan, interrompu par deux petits lacs, pour passer du lac Albanel au lac Mistassini. Deuxième hypothèse. Le 3 septembre, jour où l'on a parcouru une cinquantaine de milles, Michaux fait la traversée du lac Albanel par un temps pluvieux et vient camper près de la décharge du lac, sans s'apercevoir que le lac s'étend loin vers l'est. Ceci n'a rien de purement hypothétique: la mauvaise visibilité peut empêcher de découvrir de grandes étendues d'eau. Dans ce cas, Michaux aurait oublié de mentionner le portage entre la Nestowkanow et le lac Albanel, ce qui n'est pas inattendu, car il n'en signale aucun ce jour-là. En effet, il est bien difficile d'admettre qu'une course d'une cinquantaine de milles à la tête d'une rivière soit dépourvue de portages. Michaux n'a peut-être pas fait le court portage entre la baie Michel-Sarrazin et la baie des Renoncules, car il ne s'y trouve aucun rapide, mais un fort courant, obligeant de portager, lorsqu'on passe du lac Mistassini au lac Albanel, mais pas nécessairement dans le sens inverse. Les trois portages de Michaux seraient alors les trois secteurs du grand portage d'Opitchouan, d'environ deux ou trois milles de long, qui permettent d'éviter la décharge impassable du lac Albanel, un courant impétueux entre des murailles rocheuses. - J'incline en faveur de la seconde hypothèse. Suivant la première, Michaux aurait fait dans la journée environ 69 milles: au moins 15 milles de la Nestowkanow a l'embouchure de la décharge du lac Albanel, 36 milles sur le lac Mistassini et 18 milles sur la rivière Rupert. Même si nous réduisons 
au minimum les dernières distances mentionnées par Michaux, 12 et 6 lieues, - le trajet ne peut être inférieur à 57 milles. D'après la seconde hypothèse, le trajet aurait de 44 à 57 milles. - Rendu à la fin du portage d'Opitchouan, dans la baie Crépieul, à l'endroit où Jolliet se bâtit une maison (la maison françoise de la carte), il reprend sa course en canot vers le nord, passe par le Grand-percé, - détroit situé entre l'île Tchapahipan et la grande pointe Washimiskow, de là se rend à la pointe de la Mikoassas, puis longe la rive nord-ouest du lac Mistassini jusqu'à la Rupert. De la fin du portage d'Opitchouan à Akopitoun, ou début de la Rupert, le trajet est de 30 à 36 milles. Enfin, lorsque Michaux mentionne qu'jl a "campé sur la rive gauche N. Ouest à 6 lieues de distance du lac "), cela signifie qu'il a campé sur la Rupert, à dix-huit milles d'Akopitoun; mais cette dernière distance pourrait être moindre, même ne pas excéder douze milles.

5 septembre. "Le 5 fait environ 8 a 10 lieues et diné sur la riv. des Goelands a 16 lieues de distance du Lac... Le soir campé... 22 lieues en totalité. Campé pres la rivierre Atchouke (Riv. des. Loupsmarins). )

Comme Michaux se dirige vers la baie d'Hudson, il s'engage sans doute dans le bras de l'ouest de la Rupert, le Natastan chipi des Mistassins. La branche de l'est sert plutôt aux voyageurs se rendant vers la Eastmain, à Neokweskau notamment. En acceptant les distances maximums de Michaux, il aurait dîné (entendons par là le repas du midi) sur la Rupert, dans le voisinage du lac Miskittenau, vers $74^{\circ} 20^{\prime}$ long. W. et $51^{\circ} 12^{\prime}$ lat. N. - Les 22 lieues en totalité représentent-ils le trajet de la journée ou celui depuis le lac Mistassini ? J'opine en faveur de la première hypothèse, car le lendemain, Michaux affirme que le lac est à 26 lieues. 66 milles dans une journée est quelque chose d'extraordinaire, mais les voyageurs sont pressés par le froid ! A 26 ou 28 lieues du lac, Michaux aurait atteint les environs du $75^{\circ}$, soit sur la rivière à la Martre, soit sur la Rupert proprement dite. Ces distances toutefois ont probablement besoin d'être réduites. Il semble bien que la rivière des Goélands et la rivière Atchouke soient des bras de la Rupert, mais je n'ai pu encore les localiser avec précision. Michaux ne mentionne pas le nom Rupert, déjà connụ des Français antérieurement, puisqu'il est sur les cartes de Laure en 1731. Nous voyons d'autre 
part dans le journal du retour que l'ensemble de la rivière porte pour lui le nom de rivière des Nids de Goéland.

6 septembre. "Le 6 revemu a Mistassin. 26 lieues. Collines des environs du lac Mistassini: Sol est un sable pur a la surface et pl.bas sable argilleux. Les Pierres et les Roches sont de Quartz impur melê d'argille qqfois deterre vegetabe. Les Pierres du rivage usées par lagitation des flots présentent des formes extraordinairess parceq. les couches dargilles ou dautres substances moins dures sont plus usées... Je nay point vu des Pierres calcaires... La Décharge des Eaux de ce Lac est vers le Nord et le No d Ouest par differentes Riv. qui vont a la Baye dhudson. Lon peut y aller en 4 jours mais il faudrait 10 jo. po. revenir. Notre course fut de 28 lieues tant les Sauvages avoient envie de revenir. .)

Ce texte appelle plusieurs commentaires. 1) Quand Michaux écrit: " Revenu à Mistassin 26 lieues " ceci signifie sans doute qu'il a fait 26 lieues pour revenir à Akopitoun (le début de la décharge) de son campement le plus éloigné sur la Rupert. L'indication d'un trajet total de 28 lieues laisse croire qu'au retour les canots ont fait une couple de lieues sur la lac Mistassini. Les voyageurs auraient donc campé entre la baie Fafard et la baie Wiyachimiskau. Cette dernière (vers $73^{\circ} 28^{\prime}$ long. W.) est un excellent site de campement parce que la berge sablonneuse est bien égouttée. C'est sans doute à cet endroit, où je suis moi-même passé, que Michaux fait allusion en parlant de collines de sable pur, puisque c'est le seul point où l'auteur a pu en rencontrer au voisinage du lac. 2) Une course de 26 à 28 lieues n'est possible qu'au retour d'un voyage, lorsque le froid menace, lorsqu'il faut gagner rapidement le terme avant que l'automne gèle les cours d'eau. 3) La " décharge des eaux est par différentes rivières ": dans la première partie de son parcours, la Rupert comprend deux ou trois cours différents et très enchevêtrés. 4) ( Je n'ai point vu de pierres calcaires. " Affirmation des plus extraordinaires, à première vue, car le lit du lac Mistassini est une cuvette calcaire. Mais à y regarder de près, l'affirmation de Michaux est non seulement plausible, mais la seule possible dans les conditions du voyage. En effet, sur la rive nordouest du lac Mistassini, que longe d'abord le botaniste, on ne voit pas de rochers, sauf quelques pauvres affleurements à la surface de l'eau. Depuis la Rupert, jusqu'à une couple de milles à l'est de la baie Wiyachimiskau, ces rochers sont quartzeux. Puis apparaissent les calcaires, à la surface de l'eau également. Quelques cailloux seulement 
sont visibles, car la forêt se rend jusqu'à la ligne de rivage. Un demimille à l'ouest de l'embouchure de la Kanapétéowapouchtikemawi (vers $73^{\circ} 25^{\prime}$ ) se montrent de beaux blocs irrégulièrement usés par l'eau, phénomène qui a frappé Michaux. Ces calcaires dolomit ques, à quelques centaines de pieds de distance, peuvent sembler des gneiss ou d'autres roches du genre. Dans la traversée du lac à l'est, on ne peut voir de tables et de falaises calcaires qu'au nord de Pahipanouk et dans l'anse MacKenzie, près de l'extrémité nord-est de Tchapahipan. Ces rochers, comme la plupart des calcaires dolomitiques, ont une teinte rouille, et à moins de s'y arrêter, il n'est pas facile de les identifier. Depuis le départ du lac Saint-Jean, Michaux n'a toujours vu que des granits et des gneiss: rien de surprenant donc qu'il confonde avec eux les calcaires dolomitiques du lac Mistassini. Le voyage se faisait si vite qu'il n'y avait d'arrêts que pour le dîner et le campement $d_{1}$ soir. A la vérité, il n'y a que la journée du 7 qu'il pouvait rencontrer des affleurements calcaires, vers le milieu de la journée, à condition d'arrêter pour dîner à cet endroit précis.

7 soptembre. "Nous sommes partis de Mistassin et nous avons couru environ dix sept a dix huit lieues a cause des courans des Rivieres tres rapides... Nous avons éte en route de puis 6 heures du matin jusqua env. 6 h. du soir malgré le brouilla d et le froid. ")

Si Michaux a campé près des baies Fafard et Wiyachimiskau, comme je le crois, cette route de 18 lieues conduirait les voyageurs à la Nestowkanow à 5 ou 10 milles de la Témiscamie.

8 septembre. "Gelée a glace dans un Vase de fer blanc... A 24 lieues environ de Mistassin aupr d'une savanne... Nous avons fait environ 20 lienes av. sautés plusieurs rapides avec le couran au lieu qu'en montant nous avons été obligé de faire Portage. ")

C'est-à-dire, " jusqu'au lieu où, au voyage d'aller, nous avons fait portage ", donc jusqu'au portage de trois quarts de lieue du 2 septembre précédent. Le trajet d'aujourd'hui répète en partie celui des 2 et 3 septembre précédent.

9 septembre. "Le Dimanche 9 septembre nous avons passéle Lac des Cygnes. Nous Sommes venu coucher sur la Montagne Monte a peine. Notre course fut évaluée a 20 lieues... Notre course fut d'environ 30 lieues. )

56. Dans Sargent: “ 20 lieues au travers de plusieurs rapides ». 
Trajet, mais à rebours, des 1 er septembre, 31,30 et 29 août. Le premier septembre, la course était de 6 lieues, le 30 août, de 3 à 4 lieues, et le 31 , un trajet non évalué. - Il y a contradiction flagrante entre le début et la fin du paragraphe, séparé par une page de texte. Le premier chiffre mentionné n'est peut être pas " 20 lieues ", car il y a eu correction et l'interprétation du zéro est sujette à caution. Il semble cependant plus vraisemblable que l'auteur ait fait 20 lieues que 30 en une seule journée!

10 septembre. "Le 10 nous avons eu sur le sommet de la Montagne une Gelée blanche tre sorte et dans les ruisseaux sur lesquels l'eau passoit etoient chargés de glaçons... Nous avons fait environ 22 lieues. )"

Traiet des 28,27 et 26 août derniers.

11 septembre. "Vers nouf heures nous nous sommes embarqué. Nous avons descendu plusi rapides sans faire Portage et apres avoir fait 15 li. nous sommes arrivé aux Grands Rapides. Ici commence le Potentilla tridentata. Trois lienes audessous des Larges Rapides l'on voit des frếnes et des Ormes. Il est a remarquer quel'on n'en voit aucun depuis les Larges Rapides jusqua Mistassin. Quatre lieues au dessous des grands Rapides je vis le 1er Pinus Strobuscar je [n]'avais pas rencontré un Seul depuis cet endroit jusqu'à Mistass tant en montant quen descendant... Nous avons fart environ 14 lieues at nous avons campé aupres des premiers Pins de Weimouth (Pinus Strobus) qui se rencontrent en descendant de Mistassin. )

Trajet des 25 et 24 août derniers. L'écriture s'empâte, on sent que Michaux a les doigts gourds.

12 septembre. "Nous sommes arrivé vers 7 heures du soir au Poste du Lac St Jean et nous avons fait environ 15 lieues. .)

15 septembre. "Parti du poste sur le lac St Jean. A la distance d'une lieue et $1 / 2$ il y a une petite rivière qui tombe dans le lac. 》)

Quatre milles et demi environ à l'est de l'ancien poste de Métabetchouan (aujourd'hui Desbiens), se trouve l'embouchure de la rivière Koushpaiganish, emplacement du village moderne de Saint-Jérôme. Le reste du voyage de Michaux ne pose pas de problème géographique particulier. 


\section{Conclusions}

Le voyage, on l'a vu, ne pose réellement pas de difficiles problèmes. Il s'interprète presque à la lettre, si l'on apporte parfois des corrections aux distances. Quoi de surprenant? Pour celui qui voyage à l'aviron dans le dédale des lacs et rivières de la Province, la carte paraît toujours en défaut. On dirait qu'elle est faite pour ramener nos trajets à d'humbles proportions. Bien étonnant que Michaux ne se soit pas trompé davantage, à une époque où il n'existait aucune carte précise. Michaux est bien passé par les rivières Mistassini et Nestcwkanow, comme autrefois Jolliet; mais contrairement à ce dernier, son trajet dans les deux sens empruntait la même rivière. Grâce aux faits acquis au cours de ce voyage, nous pourrons mieux interpréter, le cas échéant, les routes de Louis Jolliet et du père Albanel. ${ }^{57}$

\section{Jacques Rousseau}

Jardin botanique de Montréal.

57. L'auteur tient à remercier ceux qui l'ont ajdé de quelque façon dans la rédaction de ce travail, notamment, son épouse, l'abbé Victor Tremblay, Madame Béatrice Grenier Bergeron, Mlles Simone Constant et Suzanne de Martigny, MM. Marcel Raymond, Ernest Rouleau, Raymond Gascon et Isaie Nantais. 